\title{
Towards an Auto-Associative Topology State Estimator
}

\author{
Jakov Krstulovic, Student Member, IEEE, Vladimiro Miranda, Fellow, IEEE, \\ Antonio J. A. Simões Costa, Fellow, IEEE, and Jorge Pereira
}

\begin{abstract}
This paper presents a model for breaker status identification and power system topology estimation based on a mosaic of local auto-associative neural networks. The approach extracts information from values of the analog electric variables and allows the recovery of missing sensor signals or the correction of erroneous data about breaker status. The results are confirmed by extensive tests conducted on an IEEE benchmark network.
\end{abstract}

Index Terms-Autoencoders, neural networks, power system topology, state estimation.

\section{INTRODUCTION}

$\mathbf{T}$ HE state estimation (SE) of a power system has been traditionally seen as the process to estimate the values of the variables of electrical nature (state variables) that characterize a given operation point, given a set of measurements affected by random errors. The classical solution for this problem takes the form of a mathematical regression in the measurement space. Also, it assumes that the topology of the network has to be defined a priori in order for the state estimation algorithm to be applied. The topology is basically defined by the status of breakers or switches, which are binary variables (open or closed). As any information coming from the SCADA system into the Energy Management System (EMS), breaker status data are also prone to be missing. In this event, heuristic rules (such as checking the power flow of an associated network branch) are often adopted as a means to restore the absent value. Alternatively, breaker status discovery may be attempted through specific topology estimation procedures.

This paper reports the advances in building a topology state estimation (TSE) procedure based on a paradigm that deviates from the classical approach. It is obvious that the topology imposes conditions on the electrical behavior of the system. Therefore, one formulates the conjecture that information about the

Manuscript received August 06, 2012; revised November 05, 2012; accepted December 21, 2012. Date of publication January 22, 2013; date of current version July 18, 2013. This work was supported in part by the ERDF from the EU through the Programme COMPETE and by the Portuguese Government through FCT-Foundation for Science and Technology, project ref. LASCA PTDC/ EEA-EEL/104278/2008 and GEMS PTDC/EEA-EEL/105261/2008. Paper no. TPWRS-00920-2012.

J. Krstulovic is with the INESC TEC (INESC Technology and Science, coordinated by INESC Porto), Porto, Portugal; with the FEUP, Faculty of Engineering of the University of Porto, Porto, Portugal; and with the University of Split, Split, Croatia (e-mail: jopara@inescporto.pt).

V. Miranda and J. Pereira are with INESC TEC (INESC Technology and Science, coordinated by INESC Porto), Porto, Portugal, and also with the FEUP, Faculty of Engineering of the University of Porto, Porto, Portugal (e-mail: vmiranda@inescporto.pt; jpereira@inescporto.pt).

A. J. A. Simões Costa is with the Federal University of Santa Catarina, Florianópolis, Brazil (e-mail: simoes@ieee.org).

Color versions of one or more of the figures in this paper are available online at http://ieeexplore.ieee.org.

Digital Object Identifier 10.1109/TPWRS.2012.2236656 topology lays hidden or disseminated in the values of the analog electric variables. A process of information extraction aimed at topology learning may therefore be conceived to reveal such distributed information: the paradigm is shifted to Information Theory, as opposed to the conventional mathematical programming.

The approach adopted in this paper is based on auto-associative neural networks (AANN) or autoencoders, trained to learn specific data manifolds, implicit in the dependence of the power flow equations on switch statuses. The feasibility of this process has already been demonstrated [1] but its full potential has not been completely explored. This paper reports the following advances:

- Establishment of the decentralized auto-associative topology processor concept based on a mosaic of local estimators of breaker status as opposed to a centralized, global estimation process;

- A new concept for a local estimator based on a competitive auto-associative neural network principle;

- The successful results of applying the competitive auto-associative solution to complex substation internal topologies ("split bus" case);

- More efficient and less computationally demanding, decentralized topology estimator, scalable to large scale systems.

These results are seen as essential building blocks for a true topology state estimator, in a process independent of the classical state estimation procedures for electrical values.

This paper is organized as follows. The problem of network topology determination is presented through a brief literature review and the new viewpoints in Section II. Section III discusses the paradigm of using AANN for missing signal restoration. Referring to the approach proposed in [1], Section IV introduces new concepts which allow the development of a more efficient and applicable topology state estimator. Section V presents comparative results based on several case studies, as well as an assessment of the proposed method performance in the presence of gross errors and reduced observability conditions. The applicability to the key "split bus" problem is evaluated in Section VI. Finally, concluding remarks and future perspectives are discussed in Section VII.

\section{Network Topology Determination}

\section{A. Past Work: Brief Review}

This section is not meant to be a full literature review but rather a highlight of distinct approaches to address the problems of topology determination and the inclusion of topology as part of the state estimation procedure. One of the first works to address those issues led to the generalized state estimation concept [2]. It is not a single model, but a series of procedures in- 
cluding a form of modeling the switching branches through null voltage difference across their terminals or null flow through them, thus allowing the inclusion of their statuses in a weighted least squares (WLS) model to estimate the electrical values. The topology determination is performed in the context of bad data identification. Hence, the methodology does not actually constitute a fully integrated topology and state estimation procedure.

Another significant contribution to a generalization of state estimation relies on the idea to define switching branch status as equality constraints and associate them to Lagrange multipliers [3]. A priori information is also included in order to avoid problems regarding critical sets of status information and system splitting during topology error identification. Based on this paradigm, the same authors developed improved methods for topology error identification [4], [5], achieving substantial reduction of computational burden. A different approach can be found in [6], where a single model is used to simultaneously perform the estimation of analog state variables and binary variables related to the network topology. A fuzzy controller is needed to adaptively tune weights and allow the correct convergence of a WLS model.

Specific topology estimation procedures have also been proposed, such as in [7]. The recent work reported in [8] is a true topology estimator model which attempts to find out the switching branch statuses from analog electrical measurements. It also relaxes the binary variables representing switches to the $[0,1]$ interval, but includes them as linearized constraints to the SE problem. The problem is then solved for these variables only as a TSE problem in the WLS sense.

As for unconventional approaches, the capabilities of artificial neural networks for topology identification have been early recognized in [9]-[11], where several methods are proposed. Such approach has been further developed in [12] and [13]. In [14], topology identification and gross error detection are performed using fuzzy pattern matching.

\section{B. A New Point of View}

TSE conventional approaches are based on mathematical programming and require the solution of an optimization problem. A new point of view, advocated in this paper, relates to pattern recognition and information theory. The rationale behind this can be summarized as follows. The Kirchhoff Laws define a manifold in the space of the electrical variables, which is the domain of the feasible solutions. The shape of this manifold changes with the state of the binary variables related to the topology (i.e., a different topology implies a different solution set) - this is why the values of the topology variables are "hidden" (or, equivalently, their information is diffusely included) in the analog electrical variables. Thus, distinct manifolds are associated to distinct topologies.

The adequate training of an AANN enables it to learn the manifold linked to a given topology. Later, when a pattern of electric values is presented to the AANN, it will "resonate" only if the pattern matches the learned manifold. In addition, if some measurement is missing in an input set of electric values, it is possible to reconstruct the missing value so that the complete pattern resonates with the learned topology.

The effect of a breaker on electric variables is mainly local: one may thus build an AANN based on local variables. This leads to another attractive property of the proposed topology processor: the fact that it is compliant with the current trend towards distributed SE conducted at the substation level [15],

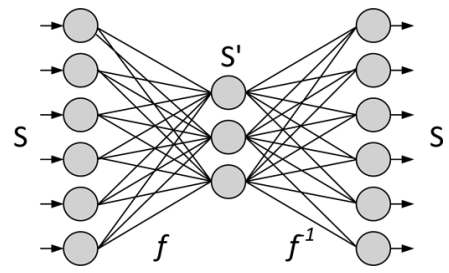

Fig. 1. Architecture of a 6-3-6 autoencoder with a single hidden layer.

[16]. In fact, the local autoencoder concept, to be discussed in Section IV, makes the auto-associative approach conformable with decentralized estimation architectures. The adoption of such architectures is seen as an important component to the evolvement of SE towards an effective integration with recent smart grids concepts [17].

\section{AuTOENCODERS}

Auto-associative neural networks or autoencoders are feedforward networks that should mirror the input space $S$ in their output. Therefore, such a network has an input vector of the same size as the output and is trained to display an output equal to its input. A trained autoencoder stores in its weights information about the training data manifold. Fig. 1 shows a simple neural network with a smaller single middle layer. This simple architecture is adopted because networks with more hidden layers have proved to be difficult to train [18], although allowing increasing accuracy.

Autoencoders with thousands of inputs have been proposed for data or image compression, using the signals available in the middle layer, which maps the input to a reduced dimension space. Reconstruction is then performed using the second half of the autoencoder (see [19], for instance). Autoencoders with neuron linear activation functions perform a feature reduction on the Principal Component sense; in this paper, nonlinear activation functions are used.

A different application is the reconstruction of missing sensor signals [20], [21]. First, a trained AANN is required. Then, if an incomplete pattern is presented to the AANN with missing components replaced by random values, a significant error $\epsilon$ will appear between the input and the output vectors. A search may then be conducted by an optimization algorithm to discover the values that should be input in the missing components such that an error $\epsilon$ becomes minimized. In a process denoted as constrained search, the optimization criterion is a function of the complete input-output vector difference, or error $\epsilon$. Any suitable optimization procedure may be used: for instance, in [22] a genetic algorithm is employed, while in [1] an evolutionary particle swarm method (EPSO) is used. In publication [1], some more information on autoencoder applications can be found, as well as references to the still scarce applications of AANN to power systems.

\section{Method CONCEPTUALIZATION}

\section{A. Global versus Local}

Recognizing that information about breaker status has strong local properties [2], the development of the method proposed in [1] has evolved towards the concept of a mosaic of local autoencoders. A local autoencoder is understood as an AANN trained to recognize the status of a breaker based in a reduced set of data. In contrast, a global autoencoder uses the whole set 


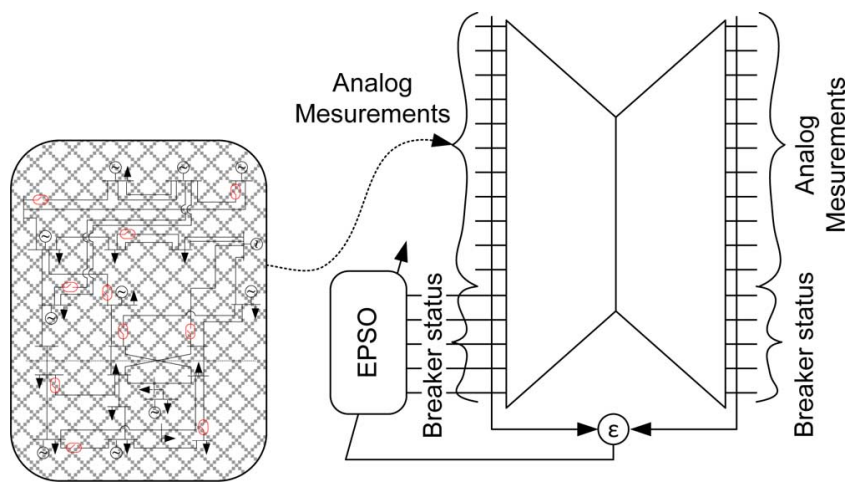

Fig. 2. Illustration of a global autoencoder-ESPO searches for the missing input values that minimize the input/output error on all the signals.

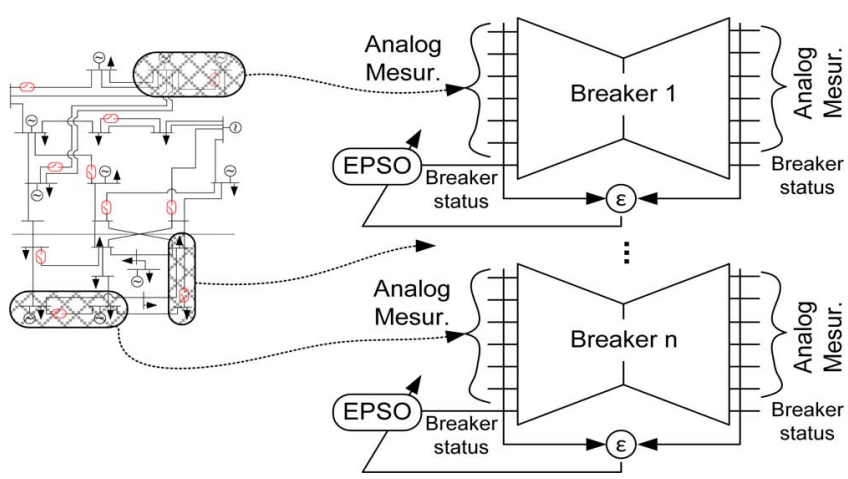

Fig. 3. Illustration of self-tuning local autoencoders - each breaker status is estimated by a specific autoencoder plus an optimization process for the missing signal.

of measurements available at the SCADA to perform breaker status recognition and, in theory, it is supposed to address the status of all breakers in the system. Figs. 2 and 3 illustrate the two approaches.

The local autoencoder has the advantage over the global autoencoder of a much smaller size, meaning that the training effort is dramatically reduced - and the training accuracy may be considerably improved. It benefits from the fact that the information on a breaker status is mostly reflected in local bus voltages and line flows.

In [1] one finds a hint that a global autoencoder could marginally outperform a local autoencoder model, particularly in cases of a high number of missing signals. With the new approach, this conclusion is totally reversed. To further look into this, in Section $\mathrm{V}$ the performance of a global autoencoder is compared with a mosaic of local autoencoders, as both strategies are applied to the same test system.

\section{B. Self-Tuning and Competitive Local Autoencoder Concepts}

The method for recognizing unknown breaker status using AANN is founded in [1], addressing the application of a constrained search (using EPSO) to determine the values of missing signals. This method will be referred to as self-tuning.

A novel, alternative local auto-associative architecture, called competitive, is introduced in this paper, inspired by the good results obtained with the technique reported in [23] for transformer fault diagnosis. The topology reconstruction is seen as

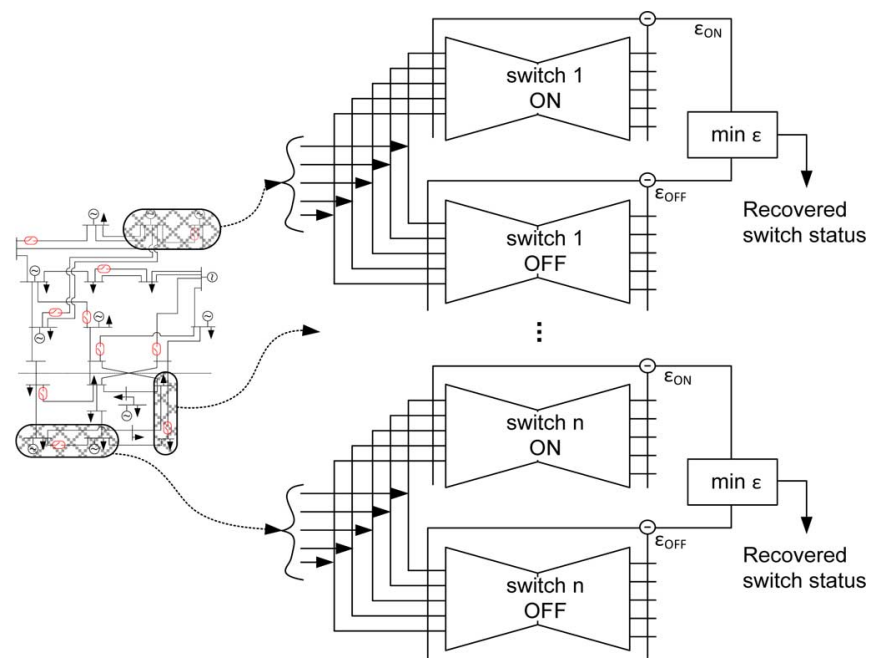

Fig. 4. Illustration of competitive local autoencoders-local signals around each breaker are input to two autoencoders causing one of them to generate a similar response, thus identifying the status.

pattern recognition problem in which each topology is associated to a particular cluster of the measurement dataset. An autoencoder is then trained for each particular cluster of data, which corresponds to a particular topology. The recognition stage relies on the fact that only a specific autoencoder will "resonate" with an unclassified sample, while others will show a significant auto-association error. In other words, when a pattern of relevant measured data is shown to all trained autoencoders, one of them will respond with a very small error (the input vector will be "in tune" with one of the patterns learned by the autoencoders), thus indicating the unknown topology.

Moreover, the well-known poor propagation characteristics of bad data in state estimation favor the use of localized strategies [2]. Considering that the elementary topology defining device is the single switch, it makes sense to narrow down the training stage to the single switch level. In this case, each breaker becomes associated with two autoencoders: one trained with local data when the status is "closed", and the other trained for the status "open" (Fig. 4). Section V quantitatively compares the performances of the self-tuning and the competitive approaches.

\section{Auto-Associative Concepts-Comparative Tests}

\section{A. Database and Method Settings}

In this section, the performance of all previously presented auto-associative topology processor concepts are tested and comparatively analyzed. The numerical results are based on the IEEE RTS 24-bus system [24] (Fig. 5), including:

a) Insertion of breakers in 10 locations of the network and random definition of their statuses ( $2^{10}$ topologies);

b) Design of a cumulative load curve with data from[24], based on which load levels are sampled and a large variety of scenarios from valley to peak of the load curve are constructed (ratio maximum $/$ minimum power $=$ $100 \% / 34 \%$ );

c) Simulation of load variations by adding a Gaussian perturbation with standard deviation $\sigma=5 \%$; 


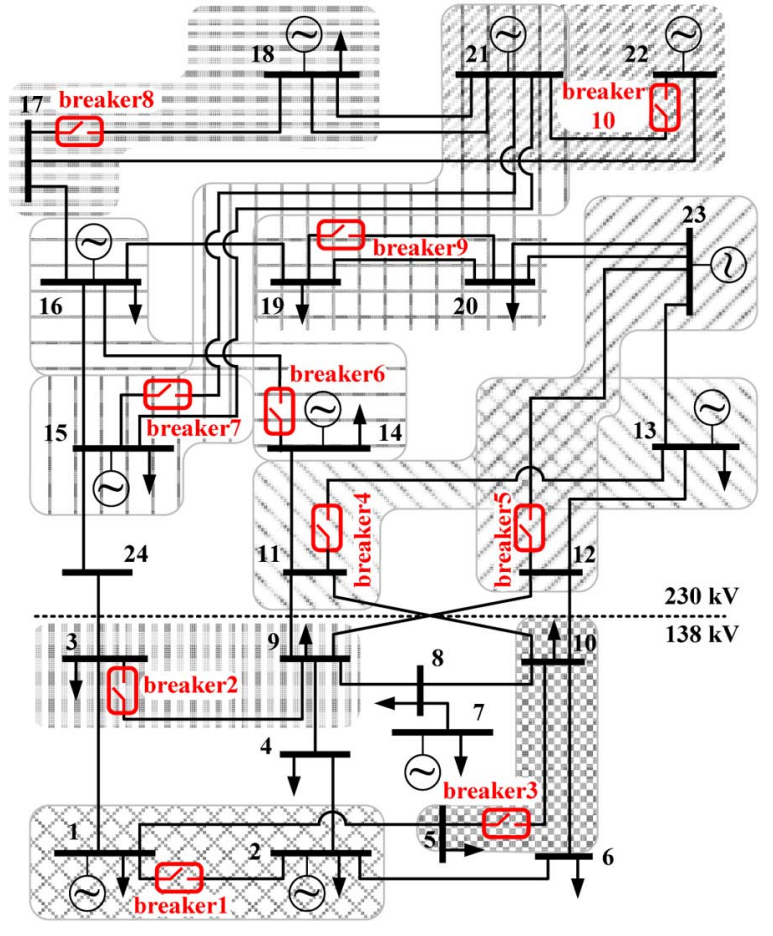

Fig. 5. IEEE RTS 24 with indication of the branches where 10 switches were introduced and the areas of measurement collection used for training 10 sets of local autoencoders.

d) Generation of a large set of power flow results by using an OPF, with breaker status randomly defined;

e) Simulation of noisy measurements by adding a Gaussian perturbation to power flow solutions, with $3 \sigma=1 \%$ or 0.01 p.u, (1 p.u. corresponds to $100 \mathrm{MVA})$.

Each autoencoder is composed of a single hidden layer. The activation functions in the input and middle layers are sigmoid and in the output layer are linear.

To compare the effectiveness of global and local models, ten distinct breaker locations in the test system are specified. For each breaker, a set of local measurements was chosen to serve as input to the AANN, as illustrated in Fig. 5. Their selection is done using engineering judgment: data from nodes and lines adjacent to the device being modeled (only active/reactive power injection and flow measurements are used). The training of the autoencoders is performed using 10000 random (breaker status+ load) scenarios.

\section{B. Missing Signal Reconstruction-Feasibility of a Mosaic of Competitive Autoencoders}

This study comprises 1000 scenarios for each case of a fixed number of 1 to 10 simultaneously unknown breaker statuses. Tables I-III summarize the statistical results related to both the correct recovery of individual breaker status and the full recognition of the network topology, for 3 alternative strategies: a global self-tuning autoencoder such as in [1], a mosaic of local self-tuning autoencoders, and a mosaic of competitive local autoencoders. In each table, "signal" refers to a single device, "topology" refers to the whole network. One wrongly estimated breaker status invalidates the reconstruction of the
TABLE I

Results From ApPlying Global Autoencoder FOR RECOMPOSING 1-10 Missing SWITCH STATUS

\begin{tabular}{|c|c|c|c||c|c|c|}
\hline \multirow{2}{*}{$\begin{array}{c}\text { No. of } \\
\text { miss. } \\
\text { signals }\end{array}$} & \multicolumn{3}{|c||}{ Signal reconstructions } & \multicolumn{2}{c|}{ Topology reconstruction } \\
\cline { 2 - 7 } & Wrong & Corr. & Efficien. & Wrong & Corr. & Efficien. \\
\hline 1 & 17 & 983 & $98.30 \%$ & 17 & 983 & $98.30 \%$ \\
\hline 2 & 28 & 1972 & $98.60 \%$ & 28 & 972 & $97.20 \%$ \\
\hline 3 & 35 & 2965 & $98.83 \%$ & 34 & 966 & $96.60 \%$ \\
\hline 4 & 51 & 3949 & $98.72 \%$ & 49 & 951 & $95.10 \%$ \\
\hline 5 & 65 & 4935 & $98.70 \%$ & 62 & 938 & $93.80 \%$ \\
\hline 6 & 84 & 5916 & $98.60 \%$ & 78 & 922 & $92.20 \%$ \\
\hline 7 & 95 & 6905 & $98.64 \%$ & 89 & 911 & $91.10 \%$ \\
\hline 8 & 121 & 7879 & $98.49 \%$ & 113 & 887 & $88.70 \%$ \\
\hline 9 & 139 & 8861 & $98.46 \%$ & 127 & 873 & $87.30 \%$ \\
\hline $\mathbf{1 0}$ & $\mathbf{1 9 5}$ & $\mathbf{9 8 0 5}$ & $\mathbf{9 8 . 0 5 \%}$ & $\mathbf{1 6 7}$ & $\mathbf{8 3 3}$ & $\mathbf{8 3 . 3 0 \%}$ \\
\hline Total & $\mathbf{8 3 0}$ & $\mathbf{5 4 1 7 0}$ & $\mathbf{9 8 . 4 9 \%}$ & $\mathbf{7 6 4}$ & $\mathbf{9 2 3 6}$ & $\mathbf{9 2 . 3 6 \%}$ \\
\hline
\end{tabular}

TABLE II

Results From Applying 10 Local Self-Tuning Autoencoders FOR RECOMPOSING 1-10 MisSing SWITCH STATUS

\begin{tabular}{|c|c|c|c||c|c|c|}
\hline \multirow{2}{*}{$\begin{array}{c}\text { No. of } \\
\text { miss. } \\
\text { signals }\end{array}$} & \multicolumn{2}{|c||}{ Signal reconstructions } & \multicolumn{2}{c|}{ Topology reconstruction } \\
\cline { 2 - 6 } & Wrong & Corr. & Efficien. & Wrong & Corr. & Efficien. \\
\hline 1 & 10 & 990 & $99.00 \%$ & 10 & 990 & $99.00 \%$ \\
\hline 2 & 17 & 1983 & $99.15 \%$ & 17 & 983 & $98.30 \%$ \\
\hline 3 & 21 & 2979 & $99.30 \%$ & 21 & 979 & $97.90 \%$ \\
\hline 4 & 37 & 3963 & $99.08 \%$ & 37 & 963 & $96.30 \%$ \\
\hline 5 & 38 & 4962 & $99.24 \%$ & 38 & 962 & $96.20 \%$ \\
\hline 6 & 46 & 5954 & $99.23 \%$ & 46 & 954 & $95.40 \%$ \\
\hline 7 & 60 & 6940 & $99.14 \%$ & 59 & 941 & $94.10 \%$ \\
\hline 8 & 71 & $\mathbf{7 9 2 9}$ & $99.11 \%$ & 68 & 932 & $93.20 \%$ \\
\hline 9 & 70 & 8930 & $99.22 \%$ & 67 & 933 & $93.30 \%$ \\
\hline $\mathbf{1 0}$ & $\mathbf{8 1}$ & $\mathbf{9 9 1 9}$ & $\mathbf{9 9 . 1 9 \%}$ & $\mathbf{7 9}$ & $\mathbf{9 2 1}$ & $\mathbf{9 2 . 1 0 \%}$ \\
\hline Total & $\mathbf{4 5 1}$ & $\mathbf{5 4 5 4 9}$ & $\mathbf{9 9 . 1 8 \%}$ & $\mathbf{4 4 2}$ & $\mathbf{9 5 5 8}$ & $\mathbf{9 5 . 5 8 \%}$ \\
\hline & & & & & & \\
\hline
\end{tabular}

TABLE III

Results From APPlying 10 Local Competitive Autoencoders FOR RECOMPOSING 1-10 Missing SWITCH STATUS

\begin{tabular}{|c|c|c|c||c|c|c|}
\hline \multirow{2}{*}{$\begin{array}{c}\text { No. of } \\
\text { miss. } \\
\text { signals }\end{array}$} & \multicolumn{2}{|c||}{ Signal reconstructions } & \multicolumn{2}{c|}{ Topology reconstruction } \\
\cline { 2 - 7 } & Wrong & Corr. & Efficien. & Wrong & Corr. & Efficien. \\
\hline 1 & 3 & 9997 & $99.97 \%$ & 3 & 9997 & $99.97 \%$ \\
\hline 2 & 5 & 19995 & $99.98 \%$ & 5 & 9995 & $99.95 \%$ \\
\hline 3 & 8 & 29992 & $99.97 \%$ & 8 & 9992 & $99.92 \%$ \\
\hline 4 & 11 & 39989 & $99.97 \%$ & 11 & 9989 & $99.89 \%$ \\
\hline 5 & 14 & 49986 & $99.97 \%$ & 14 & 9986 & $99.86 \%$ \\
\hline 6 & 15 & 59985 & $99.98 \%$ & 15 & 9985 & $99.85 \%$ \\
\hline 7 & 18 & 69982 & $99.97 \%$ & 18 & 9982 & $99.82 \%$ \\
\hline 8 & 24 & 79976 & $99.97 \%$ & 24 & 9976 & $99.76 \%$ \\
\hline 9 & 26 & 89974 & $99.97 \%$ & 26 & 9974 & $99.74 \%$ \\
\hline $\mathbf{1 0}$ & $\mathbf{2 8}$ & $\mathbf{9 9 9 7 2}$ & $\mathbf{9 9 . 9 7 \%}$ & $\mathbf{2 8}$ & $\mathbf{9 9 7 2}$ & $\mathbf{9 9 . 7 2 \%}$ \\
\hline Total & $\mathbf{1 5 2}$ & $\mathbf{5 4 9 8 4 8}$ & $\mathbf{9 9 . 9 7 \%}$ & $\mathbf{1 5 2}$ & $\mathbf{9 9 8 4 8}$ & $\mathbf{9 9 . 8 5 \%}$ \\
\hline
\end{tabular}

network topology, but a wrong topology may result from the simultaneous erroneous identification of several breakers.

It is clear that the mosaics of local autoencoders exhibit considerable superiority over the global autoencoder approach and also that they are quite insensitive to the size of the set of unknown signals. It is also apparent that the competitive model significantly outperforms the self-tuning model, especially when the number of missing signals increases. 
TABLE IV

COMPARISON OF TWO MODELS FOR LOCAL AUTOENCODER TOPOLOGY IDENTIFICATION WITH LOCAL MEASUREMENTS

\begin{tabular}{|c|c|c|c|c|c|c|c|c|}
\hline \multirow{3}{*}{ 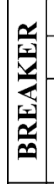 } & \multicolumn{8}{|c|}{ MODEL } \\
\hline & \multicolumn{5}{|c|}{ Self-tuning } & \multicolumn{3}{|c|}{ Competitive } \\
\hline & & 窇 & Wrong & Corr. & Efficien. & Wrong & Corr. & Efficien. \\
\hline 1 & 15 & 10 & 0 & 10000 & $100.00 \%$ & 0 & 10000 & $100.00 \%$ \\
\hline 2 & 19 & 14 & 1 & 9999 & $99.99 \%$ & 0 & 10000 & $100.00 \%$ \\
\hline 3 & 17 & 12 & 162 & 9838 & $98.38 \%$ & 17 & 9983 & $99.83 \%$ \\
\hline 4 & 17 & 12 & 0 & 10000 & $100.00 \%$ & 0 & 10000 & $100.00 \%$ \\
\hline 5 & 17 & 12 & 0 & 10000 & $100.00 \%$ & 0 & 10000 & $100.00 \%$ \\
\hline 6 & 15 & 10 & 0 & 10000 & $100.00 \%$ & 0 & 10000 & $100.00 \%$ \\
\hline 7 & 17 & 12 & 0 & 10000 & $100.00 \%$ & 0 & 10000 & $100.00 \%$ \\
\hline 8 & 13 & 8 & 381 & 9619 & $96.19 \%$ & 7 & 9993 & $99.93 \%$ \\
\hline 9 & 13 & 8 & 277 & 9723 & $97.23 \%$ & 0 & 10000 & $100.00 \%$ \\
\hline 10 & 17 & 12 & 0 & 10000 & $100.00 \%$ & 0 & 10000 & $100.00 \%$ \\
\hline \multicolumn{3}{|c|}{ Total } & 821 & 99179 & $99.18 \%$ & 24 & 99976 & $99.98 \%$ \\
\hline
\end{tabular}

\section{Identification of a Single Breaker Status-Local Autoencoders With Local Information}

In order to investigate more in detail the concepts and merits of the local autoencoder alternative models, their performance was tested reconstructing the status of each particular breaker. Table IV presents the status identification results for each of the 10 breaker locations on a sample of 10000 distinct scenarios. Those results confirm a better performance of the competitive scheme for all cases. This table includes information about the architecture of each autoencoder in the self-tuning model for each breaker location. The autoencoders in the competitive model have one neuron less in each layer, because the self-tuning AANN must account for the switch signal in the input data.

Based on these result, it is now safe to say that the competitive scheme is far superior to the self-tuning model. Plus, such strategy is also more efficient from the computing point-ofview, since it requires only input processing by the neural networks, while the self-tuning scheme requires an optimization procedure for each autoencoder.

It may also be observed that the degree of difficulty for disclosing the breaker status from the measured analog data is not the same for all breakers. The reasons behind this fact still deserve further investigation, but the problem is likely to be related to the choice of the measurement set selected as input to each autoencoder. An optimization procedure to compose such set would be a useful future addition to the technique.

\section{Impact of Input Measurements Choice-Remote Measurements Only}

An additional experiment tested the impact of the availability of local measurements. The autoencoders are now trained using a measurement set that excludes the previously used measurements. That is to say, all measurements adjacent or incident to the breakers are not employed.

Fig. 6 indicates the power flow measurements chosen for the autoencoder in charge of the breaker \#6: 14 remote measurements (active and reactive power flows) are chosen. A similar procedure is adopted to select the measurements for the other 9 switches. It is important to mention that this case does not satisfy
TABLE V

COMPARISON OF TWO MODELS FOR LOCAL AUTOENCODER TOPOLOGY IDENTIFICATION WITH REMOTE DATA ONLY

\begin{tabular}{|c|c|c|c|c|c|c|c|c|}
\hline \multirow{3}{*}{ 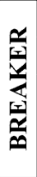 } & \multicolumn{8}{|c|}{ MODEL } \\
\hline & \multicolumn{5}{|c|}{ Self-tuning } & \multicolumn{3}{|c|}{ Competitive } \\
\hline & 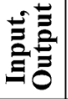 & 冚 & Wrong & Corr. & Efficien. & Wrong & Corr. & Efficien. \\
\hline 1 & 13 & 10 & 3536 & 6464 & $64.64 \%$ & 2232 & 7768 & $77.68 \%$ \\
\hline 2 & 19 & 15 & 1479 & 8521 & $85.21 \%$ & 1051 & 8949 & $89.49 \%$ \\
\hline 3 & 21 & 17 & 1837 & 8163 & $81.63 \%$ & 1235 & 8765 & $87.65 \%$ \\
\hline 4 & 19 & 15 & 1604 & 8396 & $83.96 \%$ & 286 & 9714 & $97.14 \%$ \\
\hline 5 & 15 & 12 & 3191 & 6809 & $68.09 \%$ & 2504 & 7496 & $74.96 \%$ \\
\hline 6 & 15 & 12 & 19 & 9981 & $99.81 \%$ & $\mathbf{0}$ & 10000 & $100.00 \%$ \\
\hline 7 & 13 & 10 & 3244 & 6756 & $67.56 \%$ & 2697 & 7303 & $73.03 \%$ \\
\hline 8 & 11 & 9 & 1474 & 8526 & $85.26 \%$ & 468 & 9532 & $95.32 \%$ \\
\hline 9 & 13 & 10 & 4668 & 5332 & $53.32 \%$ & 4449 & 5551 & $55.51 \%$ \\
\hline 10 & 11 & 9 & 168 & 9832 & $98.32 \%$ & 119 & 9881 & $98.81 \%$ \\
\hline \multicolumn{3}{|c|}{ Total } & 21220 & 78780 & $78.78 \%$ & 15041 & 84959 & $84.96 \%$ \\
\hline
\end{tabular}

observability requirements, which prevents the use of heuristic rules or a posteriori methods to process the breaker status.

The results in Table $\mathrm{V}$ again show that the competitive scheme performs better than the self-tuning model. Furthermore, it seems remarkable that even if surrounding information is missing, the scheme achieves a successful recovery in a large number of cases.

The results also reveal a very important hint about the choice of input measurements, namely, information about breaker status is spread throughout the analog measurements, and depends on the system parameters and topology. Although in this experiment one has removed from the input set the local measurements that were likely to contain the highest density of information about the breaker status, "shadows" of that information are still present in a "second layer" of data, more remotely located with respect to the breaker.

This is particularly noticeable in the case of breaker \#6, for which a trained autoencoder remains accurate in uncovering the breaker status. This property can be used in a backup scheme in case local information is missing, and indicates that the method is applicable even in cases of low local measurement redundancy.

\section{E. Robustness to Gross Errors}

The performance of the local competitive autoencoder mosaic has been also submitted to a stress test in the presence of gross errors (GE) in the measurement set. The topology estimator efficiency is evaluated for each particular autoencoder by contaminating its input data in 10000 scenarios with 1 to 5 randomly generated gross errors whose variances are equal to $30 \sigma$ (30 times the noise previously added to the "exact values"- errors below $10 \sigma$ are not considered). A remarkable robustness emerged, as the results in Table VI show: with a radical stress test of 5 simultaneous gross errors in the data from $25 \%$ to $50 \%$ of the inputs, depending on the autoencoder, the topology recognition for most of the 10 breaker status missing remains above $80 \%$. The lower efficiency in breaker 8 is hampered by the fact that 5 gross errors amount to $50 \%$ of the inputs being contaminated.

Of course, this is an extreme test, because the probability of having such a high number of gross errors affecting a set of local measurements is extremely low. 
TABLE VI

BREAKER STATUS IDENTIFICATION EFFICIENCY IN THE PRESENCE OF 0-5 GROSS ERRORS

\begin{tabular}{|c|c|c|c|c|c|c|}
\hline \multirow{3}{*}{$\begin{array}{l}\frac{x}{x} \\
\frac{1}{4} \\
\frac{x}{x}\end{array}$} & \multicolumn{6}{|c|}{ Number of GE } \\
\hline & $\mathbf{0}$ & 1 & 2 & 3 & 4 & 5 \\
\hline & \multicolumn{6}{|c|}{ Breaker reconstruction efficiency [\%] } \\
\hline 1 & $100.00 \%$ & $99.75 \%$ & $99.33 \%$ & $98.29 \%$ & $97.81 \%$ & $97.05 \%$ \\
\hline 2 & $100.00 \%$ & $97.63 \%$ & $95.69 \%$ & $93.92 \%$ & $92.55 \%$ & $91.25 \%$ \\
\hline 3 & $99.83 \%$ & $93.42 \%$ & $88.57 \%$ & $85.08 \%$ & $81.85 \%$ & $80.85 \%$ \\
\hline 4 & $100.00 \%$ & $95.81 \%$ & $91.26 \%$ & $87.71 \%$ & $82.73 \%$ & $80.01 \%$ \\
\hline 5 & $100.00 \%$ & $95.57 \%$ & $91.50 \%$ & $87.69 \%$ & $84.79 \%$ & $80.41 \%$ \\
\hline 6 & $100.00 \%$ & $100.00 \%$ & $100.00 \%$ & $99.99 \%$ & $99.99 \%$ & $99.99 \%$ \\
\hline 7 & $100.00 \%$ & $99.75 \%$ & $99.51 \%$ & $98.95 \%$ & $98.72 \%$ & $98.70 \%$ \\
\hline 8 & $99.89 \%$ & $89.12 \%$ & $81.20 \%$ & $73.86 \%$ & $69.09 \%$ & $65.57 \%$ \\
\hline 9 & $100.00 \%$ & $97.66 \%$ & $95.63 \%$ & $93.69 \%$ & $91.39 \%$ & $89.49 \%$ \\
\hline 10 & $100.00 \%$ & $99.99 \%$ & $99.96 \%$ & $99.86 \%$ & $99.85 \%$ & $99.82 \%$ \\
\hline
\end{tabular}

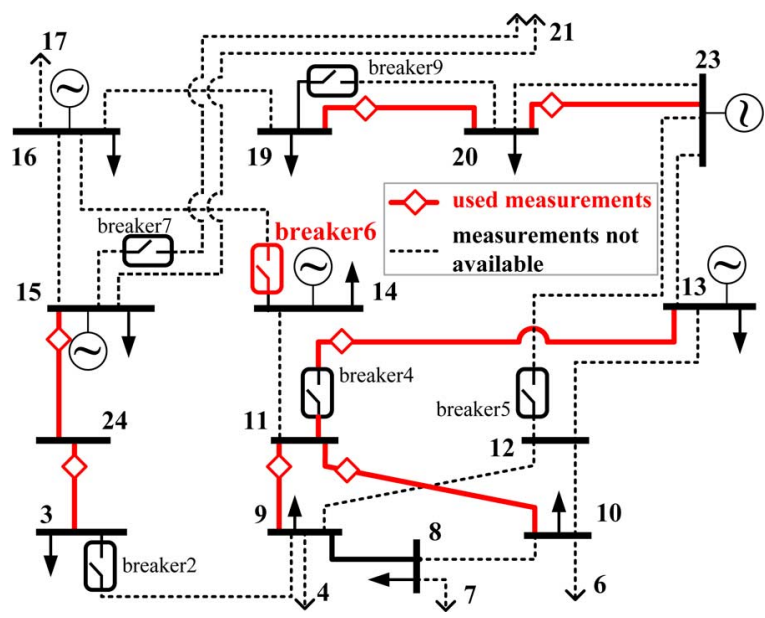

Fig. 6. Partial representation of the IEEE RTS 24 system, for the identification of breaker 6 status. Flow measurements are available only for full lines and not for dashed lines - the autoencoders can only rely on remote information.

\section{Network Topology IDENTIFICATION-Split Bus}

The term "split bus" is used in this paper to refer to the determination of the topology in complex arrangements within substations. It is generally recognized as a difficult case to model. In more precise terms, the problem is defined as follows:

- A substation is assumed as a black box;

- Only external information (measurements) is used, i.e., no measurements on switching branches are considered;

- The objective is to determine the internal substation topology (defined by sets of open and closed circuit breakers) which implies a global network topology consistent with the external measurements.

This last remark is important because there can be internal alternative connections that are undistinguishable in the sense that they define the same external connections of the network branches. Therefore, in the following we will not be concerned with the identification of individual circuit breaker status, but rather in the determination of the feasible internal substation topology.

In [1], a model for representing a complex node by an autoencoder of the self-tuning type was presented. The complex bus

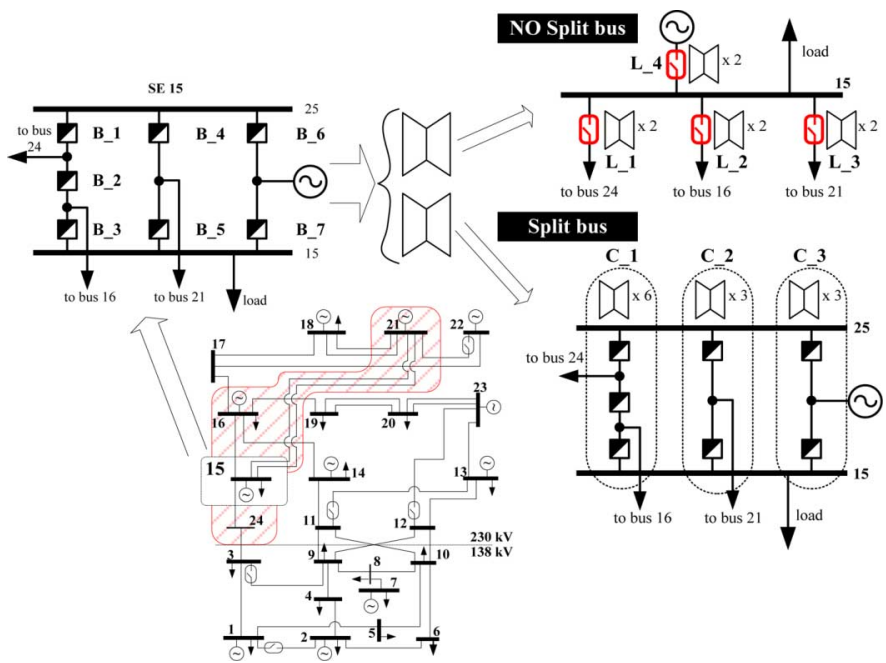

Fig. 7. Concept of local competitive autoencoders for substation topology reconstruction on the example of bus 15 .

architecture for bus 15 of the IEEE RTS 24 system and the area in which the measurements are gathered is depicted in Fig. 7.

The substation arrangement is composed of a double bus/ double breaker plus a breaker-and-a-half, and results in 47 distinguishable substation topologies. Here, "topology" stands for an electrical connection - there are several alternative ways of opening and closing breakers that result in the same connection scheme for the substation, when it is seen as a node of the transmission system. In these cases, without circuit breaker status information one may still be able to reconstruct the equivalent topology, but will be unable to specify the status of specific individual circuit breakers.

In [1], the breaker status identification is used as the basis to assess reconstruction efficiency. The model adopted was a local self-tuning autoencoder requiring 7 extra inputs for the (missing) status of the 7 breakers. In this paper, a better performing scheme based on competitive autoencoders is employed, and the results are evaluated in terms of global network topology identification.

For the substation at node 15, a new two-level strategy, illustrated in Fig. 7, is now defined to build a nodal topology estimator:

- First, a competitive autoencoder scheme is employed to decide if a split bus has occurred or not;

- Then, depending on the decision at the first level, other autoencoders are called upon to determine the status of the substation breakers.

If there is no split bus, feasible substation topologies are equivalent to 4 breakers connecting the lines and the generating source to the single bus. On the other hand, in the case the bus is split, 3 independent circuits must be considered and in each of them at least one breaker must be open. In each of the circuits with 2 breakers, only 3 cases are possible (line connected to bus 1 , bus 2 or disconnected), so that 3 autoencoders are trained individually to set up a parallel competitive arrangement. For the circuit with 3 breakers, 6 distinct topologies are possible, leading to the training of 6 autoencoders that will compete with each other when faced with an unclassified sample. Needless to say, all training is done offline. 
TABLE VII

COMPETITIVE AutOENCODERS -DetaILED RESUlts FOR THE SUbSTATION 15 TOPOlOgy RECONSTRUCTION (10 000 CASES)

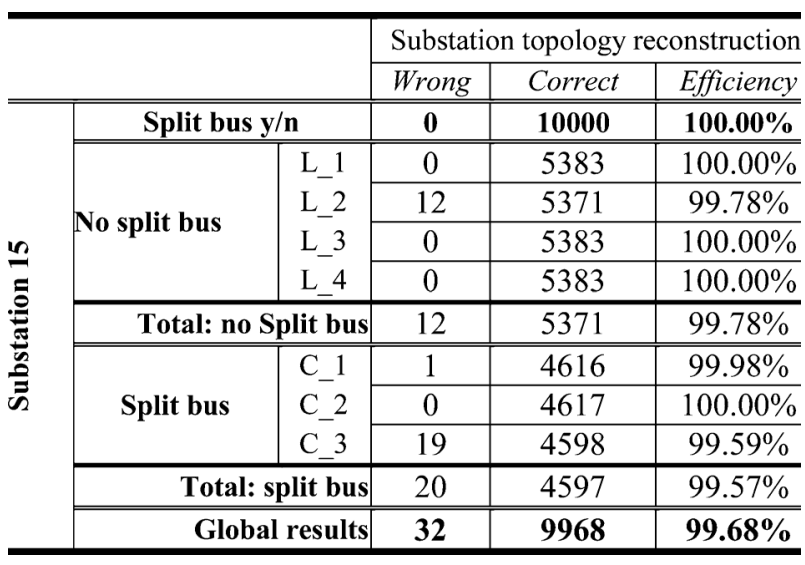

TABLE VIII

RESUltS FOR the SUbSTATION TOPOLOGY IDENTIFICATION IN THE CASE OF SUBSTATIONS $1,9,12,15$, AND 16 (10 000 CASES)

\begin{tabular}{c|c|c|c|c|c|c}
\cline { 5 - 7 } & \multicolumn{3}{c}{} & \multicolumn{3}{c}{ Subsystem topology reconstruction } \\
\hline $\begin{array}{c}\text { Sub- } \\
\text { station }\end{array}$ & $\begin{array}{c}\text { No. } \\
\text { breakers }\end{array}$ & $\begin{array}{c}\text { No. } \\
\text { AANN }\end{array}$ & $\begin{array}{c}\text { No. } \\
\text { topol. }\end{array}$ & Wrong & Correct & Efficiency \\
\hline SE 1 & 6 & 17 & 20 & 30 & 9970 & $99.70 \%$ \\
\hline SE 9 & 10 & 32 & 205 & 671 & 9329 & $93.29 \%$ \\
\hline SE 12 & 8 & 22 & 66 & 477 & 9523 & $95.23 \%$ \\
\hline SE 15 & 7 & 22 & 35 & 32 & 9968 & $99.68 \%$ \\
\hline SE 16 & $\mathbf{8}$ & $\mathbf{2 2}$ & $\mathbf{6 6}$ & $\mathbf{3 8 8}$ & $\mathbf{9 6 1 2}$ & $\mathbf{9 6 . 1 2 \%}$ \\
\hline
\end{tabular}

This strategy to build a complex node topology estimator is tested with 10000 scenarios. Only power flow and injection measurements external to the substation (from the shaded area of Fig. 7) are used.

The results are organized in Table VII. First of all, the proposed strategy reached the remarkable result of $100 \%$ correctness in identifying the yes/no existence of a split bus case, which makes a significant starting point.

In the second decision phase, the accuracy is also extremely good, in spite of no information from inside the substation having been used. In all cases, the 7 switch statuses are considered unknown, and no voltage information is assumed available.

These results, obtained in severe conditions of lack of information, are considered very promising and a significant improvement regarding the previous self-tuning model.

To confirm this assertion, Table VIII shows results for 4 more cases of entire substation topology identification. These substations are modeled with the double-bus/double-breaker circuit breakers schemes. The method remains very efficient.

\section{CONCLUSIONS}

\section{A. Lessons Learned}

This work reports the working progress in the application of auto-associative neural networks to conceive a topology state estimator. First of all, it reinforces the credibility of the hypothesis that information on breaker statuses in a power network is embedded in the values of electric analog variables of the system. The conjecture is that a given network topology constrains the solutions of the Kirchhoff Laws to a specific pattern. This being so, training an AANN to learn such a pattern becomes a feasible topology estimation strategy.

This work introduced a general model composed of a mosaic of local autoencoders, each of which is in charge of a particular "topology cell". This dissemination of localized autoassociators makes a substantial step towards system topology processing decentralization, which is particularly suitable for modern power system models.

The mapping of topologies to specific clusters in the available analog measurement dataset has led to the two principal approaches to the problem of missing signal reconstruction. Besides the self-tuning model proposed in [1], another approach, brought as a novelty in this paper, establishes a competitive auto-associative network scheme, where each autoencoder is relevant to a particular topology. The new method seems to be significantly more efficient in topology reconstruction and computing time. Moreover, the competitive approach proved to be resilient in the presence of gross analog measurement errors, even when an abnormally large number of bad data are present. The detection and identification of gross errors in analog data is not the focus of this paper but nevertheless the results suggest that this is a line to pursue.

A new scheme employing the principle of competitive autoencoders has been put in place in a complex substation design, with very encouraging results. Remarkable accuracy has been achieved in fully reconstructing inner substation connections for the case studies presented in this paper, especially if one considers that the results are obtained without using any flow, voltage or breaker status information internal to the substation.

The advantage of the topology state estimator based on autoencoders is that it does not require complex computations and is extremely fast. Therefore, it can be conceived as an accessory tool in a more complex SE application in an EMS environment. In addition, since the proposed model is based on local, low dimension autoencoders, the training procedures may be automated and demand low computing time (a few tens of seconds in an ordinary PC for one network). Furthermore, the training is done offline, and incremental changes of the power system, namely the addition of a new line or power station, will only affect a small subset of local autoencoders which must be retrained.

\section{B. Visions of the Future}

Based on the strong results presented in this paper, there is some margin for improvement in the application of the proposed techniques, with some avenues still to explore.

First, the process of autoencoder training can still be improved, particularly in regard to: 1) expansion of the ANNS to include multiple hidden layers, and 2) the metric used for autoencoder training. Concerning the latter, the minimum square error criterion is employed in this paper, but there are reasons to believe that other metrics, with deeper relation to information theory, might perform better, especially with respect to robustness to bad data.

In all experiments reported, the choice of measurements for ANN training has been based on engineering common sense, leaving unaddressed the problem of selecting the most efficient set of measurements to "feed" the autoencoders. Finally, the best combination of the proposed approach with the classical state 
estimation procedure is something that should be addressed. The straightforward application is to use the topology estimator in the first phase of a classical SE, as a preprocessing tool and, after establishing the topology, the SE may proceed into the estimation of electrical values. The authors do not advocate that the new models completely replace the conventional ones and strongly believe in the benefits of a judicious combination of both approaches.

\section{REFERENCES}

[1] V. Miranda, J. Krstulovic, H. Keko, C. Moreira, and J. Pereira, "Reconstructing missing data in state estimation with autoencoders," IEEE Trans. Power Syst., vol. 27, no. 2, pp. 604-611, May 2012.

[2] O. Alsaç, N. Vempati, B. Stott, and A. Monticelli, "Generalized state estimation," IEEE Trans. Power Syst., vol. 13, no. 3, pp. 1069-1075, Aug. 1998.

[3] K. Clements and A. S. Costa, "Topology error identification using normalized Lagrange multipliers," IEEE Trans. Power Syst., vol. 13, no. 2, pp. 347-353, May 1998.

[4] E. M. Lourenço, A. S. Costa, and K. A. Clements, "Bayesian-based hypothesis testing for topology error identification in generalized state estimation," IEEE Trans. Power Syst., vol. 19, no. 2, pp. 1206-1215, May 2004.

[5] E. M. Lourenço, A. S. Costa, K. A. Clements, and R. A. Cernev, "A topology error identification method directly based on collinearity tests," IEEE Trans. Power Syst., vol. 21, no. 4, pp. 1920-1929, Nov. 2006.

[6] J. Pereira, V. Miranda, and J. T. Saraiva, "Fuzzy control of state estimation robustness," in Proc. 14th PSCC, Seville, Spain, Jun. 2002.

[7] N. Vempati, C. Silva, O. Alsac, and B. Stott, "Topology estimation," in Proc. 2005 IEEE Power Eng. Soc. General Meeting, Jun. 12-16, 2005, vol. 1, pp. 806-810.

[8] E. Caro, A. J. Conejo, and A. Abur, "Breaker status identification," IEEE Trans. Power Syst., vol. 25, no. 2, pp. 694-702, May 2010.

[9] A. A. da Silva, V. Quintana, and G. K. H. Pang, "A pattern analysis approach for topology determination, bad data correction and missing measurement estimation in power systems," in Proc. 22nd Annu. North Amer., 1990, pp. 363-372.

[10] A. P. A. da Silva, V. H. Quintana, and G. K. H. Pang, "Neural networks for topology determination of power systems," in Proc. 1st Int. Forum Applicat. Neural Networks to Power Syst., Seattle, WA, USA, 1991, pp. 297-301.

[11] A. A. da Silva, V. Quintana, and G. Pang, "Solving data acquisition and processing problems in power systems using a pattern analysis approach," IEE Proc.-C, vol. 138, no. 4, pp. 365-376, 1991.

[12] D. V. Kumar, S. Srivastava, S. Shah, and S. Mathur, "Topology processing and static state estimation using artificial neural networks," IEE Proc.-Gen., Transm., Distrib., vol. 143, no. 1, pp. 99-105, 1996.

[13] J. Souza, A. L. da Silva, and A. P. A. da Silva, "Online topology determination and bad data suppression in power system operation using artificial neural networks," IEEE Trans. Power Syst., vol. 13, no. 3, pp. 796-803, Aug. 1998.

[14] D. Singh, J. Pandey, and D. Chauhan, "Topology identification, bad data processing, and state estimation using fuzzy pattern matching," IEEE Trans. Power Syst., vol. 20, no. 3, pp. 1570-1579, Aug. 2005.

[15] T. Yang, H. Sun, and A. Bose, "Transition to a two-level linear state estimator-Part I: Architecture,” IEEE Trans. Power Syst., vol. 26, no. 1, pp. 46-53, Feb. 2011.

[16] T. Yang, H. Sun, and A. Bose, "Transition to a two-level linear state estimator-Part II: Algorithm," IEEE Trans. Power Syst., vol. 26, no. 1, pp. 54-62, Feb. 2011.

[17] A. Bose, "Smart transmission grid applications and their supporting infrastructure," IEEE Trans. Smart Grid, vol. 1, no. 1, pp. 11-19, Jun. 2010.

[18] G. E. Hinton and R. R. Salakhutdinov, "Reducing the dimensionality of data with neural networks," Science, vol. 313, no. 5786, pp. 504-507, Jul. 2006.
[19] B. Golomb and T. Sejnowski, "Sex Recognition from Faces Using Neural Networks," in Applications of Neural Networks, A. Murray, Ed. Norwell, MA, USA: Kluwer, 1995, pp. 71-92.

[20] S. Narayanan, R. J. Marks, II, L. Vian, II, I. I. Choi, M. A. El-Sharkawi, and B. B. Thompson, "Set constraint discovery: Missing sensor data restoration using auto-associative regression machines," in Proc. Int Joint Conf. Neural Networks, IEEE World Congr. Computational Intell., Honolulu, HI, USA, May 12-17, 2002, pp. 2872-2877.

[21] B. B. Thompson, R. J. Marks, and M. A. El-Sharkawi, "On the contractive nature of autoencoders: Application to missing sensor restoration," in Proc. Int. Joint Conf. Neural Networks, Jul. 2003, vol. 4, pp. 3011-3016.

[22] M. Abdella and T. Marwala, "The use of genetic algorithms and neural networks to approximate missing data in databases," Comput. Informat., no. 24, pp. 577-589, 2005.

[23] V. Miranda, A. R. G. Castro, and S. Lima, "Diagnosing faults in power transformers with autoassociative neural networks and mean shift," IEEE Trans. Power Del., vol. 27, no. 3, pp. 1350-1357, Jul. 2012.

[24] IEEE RTS Task Force of APM Subcommittee, "IEEE reliability test system," IEEE Trans. Power App. Syst., vol. PAS-98, no. 6, pp. 2047-2054, Nov./Dec. 1979.

Jakov Krstulovic (S'10) received the graduation degree from the Faculty of Electrical Engineering and Computing, University of Zagreb, Zagreb, Croatia, in 2008. He is currently pursuing the Ph.D. degree at the Faculty of Engineering of the University of Porto (FEUP), Porto, Portugal.

Since October 2010, he has been a Junior Researcher in Power Systems Unit of INESC TEC, Portugal. He also works as a teaching assistant at the Faculty of Electrical Engineering, Mechanical Engineering and Naval Architecture, University of Split, Split, Croatia. His primary research interests are power system state estimation, computational intelligence, and large scale integration of the wind power.

Vladimiro Miranda (M'90-SM'04-F'06) received the B.Sc. and Ph.D. degrees in electrical engineering from the Faculty of Engineering of the University of Porto, Porto, Portugal (FEUP), in 1977 and 1982, respectively.

In 1981, he joined FEUP and currently holds the position of Full Professor. $\mathrm{He}$ also has been a researcher at INESC since 1985 and is currently Director at INESC Porto, the leading institution of INESC TEC-INESC Technology and Science, an advanced research network in Portugal. He has authored many papers and been responsible for many projects in areas related with the application of computational intelligence to power systems.

Antonio J. A. Simões Costa (F'06) received the Electrical Engineering degree from the Federal University of Pará, Belém, Brazil, in 1973, the M.Sc. degree in electrical engineering from the Federal University of Santa Catarina (UFSC), Florianópolis, Brazil, in 1975, and the Ph.D. degree in electrical engineering from the University of Waterloo, Waterloo, ON, Canada, in 1981.

Since 1975, he has been with UFSC's Department of Electrical Engineering. His research interests are concerned with computer methods for power systems operation and control.

Jorge Pereira received the degree in applied mathematics from the Faculty of Sciences of the University of Porto, Porto, Portugal, in 1991 and the M.Sc. and Ph.D. degrees in electrical and computer engineering from the Faculty of Engineering of the University of Porto, Porto, Portugal (FEUP), in 1995 and 2002, respectively.

In 1991 he joined INESC Porto and is presently a research manager at INESC TEC. Since 1995 he is with the Faculty of Economics of the University of Porto, Portugal, where he is currently an Assistant Professor. He has collaborated in several projects related to the development of DMS and the application of soft computing techniques to power systems namely to the state estimation problem. 